\title{
DEVOLUTIVA DOS RESULTADOS DE PESQUISA DESENVOLVIDA COM CUIDADORES FAMILIARES DE IDOSOS DEPENDENTES
}

\author{
Karla Ferraz dos Anjos \\ Universidade Federal da Bahia \\ karla.ferraz@hotmail.com
}

Rita Narriman Silva de Oliveira Boery

Universidade Estadual do Sudoeste da Bahia rboery@gmail.com

\author{
Vanessa Cruz Santos \\ Universidade Federal da Bahia \\ vanessacrus@hotmail.com \\ Eduardo Nagib Boery \\ Universidade Estadual do Sudoeste da Bahia \\ eboery@gmail.com
}

\section{Resumo}

Objetivo: relatar a devolutiva dos resultados de uma pesquisa desenvolvida com cuidadores familiares de idosos dependentes. Método: relato de experiência da apresentação e interpretação dos achados do estudo e da realização de palestras para cuidadores de idosos, enfermeiros e agentes comunitários de saúde, em dois municípios do interior do Estado da Bahia, no ano de 2014, totalizando 24 participantes. Utilizou-se como recurso visual o Datashow. Resultados: participantes citaram a importância dos profissionais das Estratégias Saúde da Família no apoio aos idosos e seus cuidadores, visto que o cuidador não tem recebido apoio social formal, e o apoio informal acontece de forma incipiente. Conclusão: a devolutiva dos resultados deste estudo possibilitou troca de saberes, diálogo sobre os achados e produção de novos conhecimentos sobre cuidados aos idosos dependentes, evidenciando assim a necessidade de compartilhamento dos resultados encontrados em pesquisas após sua realização, como compromisso do pesquisador e ação social.

Palavras-chave: Pesquisa. Cuidadores. Idoso. Pesquisadores.

\section{DEVOLUTION OF THE RESULTS OF A SURVEY DEVELOPED WITH CAREGIVERS OF DEPENDENT ELDERLY PEOPLE}

\section{Abstract}

Objective: to describe the devolution of the results of a survey conducted with family caregivers of dependent elderly people. Method: this is an experience report of the presentation and interpretation of the findings of the study and accomplishment of lectures for caregivers of elderly people, nurses and community health workers, in two cities in the State of Bahia, in 2014, totaling 24 participants. Projection screen was used as a visual resource. Results: the participants have cited the importance of the professionals of the Family Health Strategies to support the elderly people and their caregivers, since the caregiver has not received formal social support, and the informal support happens in an incipient form. Conclusion: the devolution of the results of this study has enabled the exchange of skills, dialogue about the findings and production of new knowledge about the care for the dependent elderly people, thus highlighting the need to share the results found in studies after its accomplishment, as a commitment of the researcher and a social action.

Keywords: Research. Caregivers. Elderly. Researchers.

\section{DEVOLUTIVA DE LOS RESULTADOS DE INVESTIGACIÓN DESARROLLADA CON CUIDADORES FAMILIARES DE ANCIANOS DEPENDIENTES}

Resumen

Objetivo: relatar la devolutiva de los resultados de una investigación desarrollada con cuidadores familiares de ancianos dependientes. Método: relato de experiencia de la presentación e interpretación de los hallazgos del estudio y de la realización de conferencias para cuidadores de ancianos, enfermeros y agentes comunitarios de salud, en dos ciudades del interior del Estado de Bahía, en el año 2014, totalizando 24 participantes. Se utilizó como recurso visual una pantalla de proyección. Resultados: los participantes citaron la importancia de los profesionales de las Estrategias Salud de la Familia en el apoyo a los ancianos y sus cuidadores, ya que el cuidador no ha recibido el apoyo social formal, y el apoyo informal ocurre de manera incipiente. Conclusión: la devolutiva de los resultados de este estudio posibilitó el intercambio de saberes, el diálogo sobre los hallazgos y la producción de nuevos conocimientos sobre la atención a los ancianos dependientes, lo que demuestra la necesidad de compartir los resultados encontrados en investigaciones después de su realización, como un compromiso del investigador y una acción social. 
Palabras clave: Investigación. Cuidadores. Anciano. Investigadores. 
Devolutiva dos resultados de pesquisa desenvolvida com cuidadores familiares de idosos dependentes

\section{INTRODUÇÃO}

Este estudo trata de uma experiência de devolutiva dos resultados da pesquisa 'Qualidade de vida do familiar cuidador de idosos dependentes no domicílio' (ANJOS, 2013). Esta etapa constitui parte essencial do compromisso do pesquisador após a realização de pesquisas e pode contribuir para com os participantes do estudo, neste caso os cuidadores de idosos. Isto porque ao conhecer aspectos relacionados ao cuidado do idoso e possíveis implicações que este pode ocasionar à vida do cuidador/idoso, estratégias preventivas podem ser planejadas e implementadas.

Nota-se que as pesquisas que envolvem seres humanos necessitam atender a alguns fundamentos éticos e científicos pertinentes, como o de assegurar aos participantes da pesquisa os benefícios resultantes do projeto, ou seja, o retorno social (BRASIL, 2012). É neste contexto de compromisso do pesquisador que este relato de experiência se justifica devido à preocupação que a "comunidade acadêmica" precisa ter quanto ao desenvolvimento de pesquisas, isto para que tanto os participantes do estudo, os profissionais da área da saúde e a comunidade envolvida tenham conhecimento dos resultados encontrados na pesquisa após sua realização. Isso pode favorecer a organização de ações direcionadas às demandas identificadas, assim como a motivação das pessoas colaborarem com o desenvolvimento de outras pesquisas.

O Código de Ética dos Profissionais de Enfermagem, em seu capítulo III do ensino, da pesquisa, e da produção técnico-científica dispõe como responsabilidades e deveres dos profissionais de enfermagem "disponibilizar os resultados de pesquisa à comunidade científica e sociedade em geral" (COREN-BA, 2012). Essa responsabilidade pode acontecer por meio da devolução dos resultados a partir de sua publicação, construção de cartilhas e manuais informativos, produção de livros, palestras, cursos e oficinas.

Neste estudo, os resultados foram apresentados a partir de palestras e de discussão entre pesquisadores-participantes e profissionais vinculados às Estratégias Saúde da Família (ESF) e gestores da saúde, de municípios localizados no interior da Bahia, que estão envolvidos na assistência prestada a essa clientela. Essa iniciativa é relevante, pois pode favorecer a aproximação com os cuidadores que, em várias circunstâncias, necessitam de apoio social formal para exercer o cuidado ao idoso.

A necessidade dessa devolutiva também se fez imprescindível diante dos resultados encontrados na pesquisa, dentre os quais, elevada sobrecarga entre os cuidadores e sua influência na qualidade de vida dessas pessoas, déficit de orientações para os cuidadores sobre como cuidar de idoso no domicílio, assim como inexistência de apoio social formal e déficit de apoio social 
informal (ANJOS, 2013; ANJOS, BOERY, PEREIRA, 2014). Logo, este momento de trocas de saberes, por meio do diálogo entre pesquisador, participantes do estudo e profissionais da área da saúde pode ser considerada estratégia fundamental de apoio e esclarecimento de dúvidas entre os envolvidos. Além do que, os dados da pesquisa apresentados e discutidos com os diversos atores sociais envolvidos no cuidado ao idoso podem contribuir para minimizar impactos biopsicossociais aos cuidadores.

Este relato torna-se justificável devido às lacunas existentes na produção científica nacional quando se realiza buscas de artigos produzidos no Brasil sobre experiências de pesquisadores que retornam os resultados produzidos após a concretização de suas pesquisas à comunidade. A escassez de estudos foi evidenciada, de maneira geral, em todas as áreas do conhecimento.

Entretanto, a leitura de dois artigos trouxeram contribuições para a construção do relato de pesquisa em tela por se assemelharem com esta proposta de devolutiva, o de Patzlaff e Peixoto (2009), que descreve sobre a pesquisa em etnobotânica e o retorno do conhecimento sistematizado à comunidade; e o de Pessanha, Silva e Rotenberg (2013), que trata de uma experiência de restituição de resultados em saúde do trabalhador. Estes estudos citam contribuições da devolução dos resultados como o diálogo, o compartilhamento e a troca entre os saberes, ampliação do conhecimento, compromisso ético do pesquisador, convivência, parceria e reconhecimento pela ajuda e pelo tempo despendido.

Vê-se que a devolução dos resultados da pesquisa é fundamental para a comunidade, uma vez que este momento favorece o diálogo entre os envolvidos, logo, o pesquisador necessita buscar estratégias que favoreçam esse retorno. Isso possibilita, por meio de compartilhamento de saberes, benefícios aos participantes, seja com informações, orientações ou esclarecimento de dúvidas. Neste caso a ser descrito, a partir da apresentação dos resultados alcançados, demonstração de dificuldades, facilidades e potencialidades dos cuidadores, poderá ajudá-los na condução do cuidado ao idoso e de seu autocuidado. Também poderá influenciar na atuação dos profissionais da área da saúde que estão diretamente envolvidos com essas pessoas.

Para tanto, este estudo objetiva relatar a devolutiva dos resultados de uma pesquisa desenvolvida com cuidadores familiares de idosos dependentes. 
Devolutiva dos resultados de pesquisa desenvolvida com cuidadores familiares de idosos dependentes

\section{MATERIAIS E MÉTODOS}

Relato de experiência de devolutiva dos resultados de uma pesquisa desenvolvida por enfermeiras, a partir de dois encontros com cuidadores de idosos, enfermeiros e Agentes Comunitários de Saúde (ACS), por meio de palestras, após a realização de pesquisa de mestrado acadêmico, vinculada ao Programa de Pós-Graduação em Enfermagem e Saúde da Universidade Estadual do Sudoeste da Bahia, Campus de Jequié. Os encontros foram indispensáveis para cumprir uma das etapas do compromisso do pesquisador, a de retorno e interpretação dos resultados.

Este relato é, portanto, resultado da experiência da pesquisa desenvolvida com cuidadores familiares de idosos dependentes, residentes em dois municípios do interior da Bahia - Manoel Vitorino e Jequié, por intermédio das ESF e apoio dos ACS, após a realização da pesquisa 'Qualidade de vida do familiar cuidador de idosos dependentes no domicílio' (ANJOS, 2013), aprovada pelo Comitê de Ética em Pesquisa, da Universidade Estadual do Sudoeste da Bahia, sob o protocolo n $\mathrm{n}^{\mathrm{o}}$ 128.580/2012 e CAAE: 08643612.6.0000.0055.

Os dois encontros foram desenvolvidos em janeiro de 2014, com a participação de duas enfermeiras mestrandas facilitadoras das palestras (uma autora da dissertação de mestrado e uma colaboradora da atividade) e dois professores doutores (um orientador e um colaborador da pesquisa). Quanto aos convidados que participaram da devolução dos resultados, foram 19 ACS, dois enfermeiros que trabalhavam nas ESF, um enfermeiro gestor e dois cuidadores, totalizando 24 pessoas, de ambos os sexos. Ressalta-se que mesmo a pesquisa sendo feita com cuidadores de idosos, a devolutiva dos resultados incluiu a participação desses profissionais da área da saúde devido à necessidade destes, junto com os cuidadores, de conhecerem a realidade vivenciada por familiares que disponibilizam tempo para cuidar do idoso dependente no domicílio. Essa prática, de alguma forma, beneficia o trabalho desses profissionais e o binômio idoso-cuidador.

Inicialmente, houve o convite prévio com agendamento de data, horário e local para os cuidadores dos idosos e profissionais das duas ESF dos municípios supracitados para participarem das palestras que tiveram o propósito de apresentar os principais resultados da dissertação de mestrado sobre a 'qualidade de vida de cuidadores de idosos' e a influência da sobrecarga de atividades diárias do cuidador e grau de dependência do idoso com a qualidade de vida (QV) dos cuidadores. Neste sentido, as mestrandas apresentaram aspectos positivos e negativos relacionados ao cuidado domiciliar e, a partir de então, apontaram algumas estratégias que podem ser realizadas por profissionais de saúde e ACS no apoio de cuidadores. 
Em cada encontro houve: 1) Apresentação e ambientação dos participantes, facilitadores e a justificativa da proposta de devolução dos resultados da pesquisa; 2) Apresentação dos principais resultados da pesquisa com a abordagem de estratégias que podem ser adotadas pelos profissionais das ESF como orientações sobre cuidados domiciliares aos idosos, baseados em manuais do Ministério da Saúde: a) Envelhecimento e saúde da pessoa idosa (BRASIL, 2007); b) Guia prático do cuidador (BRASIL, 2008a) e, c) Cuidar melhor e evitar a violência: manual do cuidador da pessoa idosa (BRASIL, 2008b) e; 3) Relato de cuidadores sobre o cuidado ao idoso no domicílio, assim como de enfermeiros e ACS sobre a assistência/atenção aos idosos. Em seguida, como forma de avaliação, os participantes comentaram sobre a experiência de participar das palestras e mencionaram sugestões para atividades futuras.

\section{RESULTADOS}

A devolutiva dos resultados da pesquisa desenvolvida com cuidadores familiares de idosos dependentes foi realizada nas: 1) Clínica de Atendimento em Saúde Mental - Manoel Vitorino e, 2) Estratégia Saúde da Família - Jequié. Ambos os lugares não favoreceram o desenvolvimento das palestras devido à estrutura física do local ser pequena para a quantidade de participantes, além do que estes são ambientes de constante movimentação de usuários dos serviços de saúde citados, o que propiciou interrupções durante as atividades e saída de alguns profissionais, sobretudo, de enfermeiros que eram requisitados por usuários dos serviços devido a alguma demanda, causando assim, algumas vezes, distrações. Além disso, a reduzida carga horária para os facilitadores que apresentaram os principais resultados encontrados na pesquisa não contribuiu, o que necessitaria de outros momentos, com intervalos entre os encontros, para exploração e aprofundamento dos diálogos e trocas de saberes e, assim, possibilitar maior contribuição dos envolvidos (ACS, cuidadores e enfermeiros).

Após a apresentação de cada participante (cuidadores, enfermeiros e ACS) no encontro, as enfermeiras facilitadoras a princípio fizeram algumas considerações sobre o aumento da população idosa mundialmente e no Brasil, a expectativa de vida, o processo de envelhecimento, a dependência funcional e cognitiva do idoso, bem como as demandas de cuidados de idosos. E apresentaram os principais resultados da pesquisa apontando a influência da sobrecarga do cuidador familiar e o grau de dependência do idoso à qualidade de vida desse cuidador.

As enfermeiras facilitadoras fizeram algumas orientações aos participantes sobre os cuidados aos idosos no domić́lio baseadas em manuais do Ministério da Saúde (BRASIL, 2007; 
2008a; 2008b), com destaque para os temas: 1) Relação dos cuidadores de idosos e profissionais de saúde; 2) Relevância da informação/orientação para cuidadores; 3) Responsabilidades pelos cuidados dos idosos; 4) Autocuidado do cuidador; 5) Cuidados com a alimentação, medicações e higiene do idoso; 6) Maus tratos; 7) Estímulo à autonomia do idoso e; 8) Apoio ao cuidador. Para a abordagem dessas temáticas, que aconteceu de maneira sucinta e informativa, utilizou-se como recurso o Datashow, com a demonstração de imagens disponibilizadas nos manuais supracitados. Durante a apresentação, os profissionais das ESF e cuidadores de idosos se envolveram no diálogo, com questionamentos e troca de experiências.

Houve também breve discussão com os cuidadores, enfermeiros e ACS sobre a importância dos profissionais vinculados às ESF identificarem os familiares responsáveis pelos cuidados aos idosos no domicílio, uma vez que estes podem necessitar de ajuda, tanto relacionada ao cuidado como de sua própria vida. Assim, foram apresentados alguns dos benefícios do apoio social às famílias cuidadoras, isto porque os resultados da pesquisa de mestrado (ANJOS, 2013) evidenciaram que os cuidadores não têm recebido apoio social formal, e o apoio informal ainda acontece de maneira incipiente e relacionado ao cuidado direto ao idoso. Achado preocupante, diante dos benefícios que o apoio social pode trazer para os cuidadores, como a minimização de implicações biopsicossociais associadas ao cuidado.

Posterior à explanação dos principais resultados da pesquisa, foi oportunizado momento de diálogo entre os participantes, com a finalidade de proporcionar interação entre os envolvidos e sanar possíveis dúvidas. Neste momento, obtiveram-se relatos de cuidadores, enfermeiros que trabalhavam nas ESF, enfermeiro gestor e ACS, sobre a experiência de participar dessa atividade de devolutiva dos resultados.

Mesmo que minoria dos participantes, os ‘cuidadores' informaram prazer e satisfação em desenvolver o cuidado ao idoso, ao tempo em que referem também o dever. Eles destacam que essa atividade está comprometendo sua vida social, porque sentem que estão deixando de realizar diversas atividades extras domésticas que realizavam anteriormente devido à assunção do cuidado. Relatam que também têm problemas de saúde e, algumas vezes, se cansam de cuidar. Que continuam cuidando por amor e pela necessidade e/ou por não ter outra pessoa para ajudar o idoso a desenvolver suas atividades cotidianas e que sua participação na devolução dos resultados da pesquisa é descrita como um momento de lazer, troca de experiência e apoio para realizar o cuidado ao idoso.

Os 'enfermeiros das ESF' opinaram sobre o cuidado domiciliar, destacando a necessidade dos profissionais de saúde atentar às necessidades e/ou demandas também dos cuidadores que, muitas das vezes, também são idosos. Ao tempo em que apontaram dificuldades relacionadas à 
assistência aos idosos dependentes e seus cuidadores no domicílio, desde a elevada demanda de usuários vinculados às ESF para assistir, assim como a indisponibilidade de transporte para deslocamento dos profissionais até o domicílio, o que, por sua vez, influencia na assistência à saúde prestada a essas pessoas.

O 'enfermeiro gestor’ de um dos municípios reconheceu a necessidade de maior atenção à saúde por parte, principalmente, dos profissionais das ESF para com as demandas dos cuidadores, enfatizando que os resultados encontrados na dissertação de mestrado relacionados ao adoecimento dos cuidadores podem ter relação com a possível "falha na atenção básica à saúde”. Diante do comentário, ressaltou a necessidade de ampliação de investimentos na área do envelhecimento na atenção básica, ao reconhecer que a idade é fator que influencia no adoecimento das pessoas e, consequentemente, traz consigo implicações biopsicossociais não somente para o idoso, mas, também, para a família, sobretudo para o cuidador principal o qual está mais próximo do idoso dependente. Eles referiram que a devolução dos resultados da pesquisa contribui no planejamento dos cuidados aos idosos e suas famílias.

Além disso, o gestor mencionou a relevância da devolução dos resultados da pesquisa para os envolvidos no cuidado ao idoso e município, destacando que, a partir do conhecimento dos dados identificados, a equipe de saúde poderá planejar ações capazes de beneficiar o cuidador-idoso-família, assim como reduzir gastos futuros para o sistema de saúde. Também informa que esse tipo de atividade oportuniza conhecer sobre a realidade vivenciada por cuidadores de idosos no domicílio e o que pode estar influenciando o seu adoecimento. Isso possibilita aos profissionais das ESF refletirem sobre a atenção prestada aos idosos e seus cuidadores, assim como favorece a tomada de decisão responsável junto à comunidade.

Um dos profissionais que atuam na ESF é o ACS, e por vivenciarem diversos problemas cotidianos e residirem na comunidade em que desempenham seu trabalho, os 'Agentes Comunitários de Saúde’ configuram-se como importante elo entre a equipe, o idoso e o cuidador, uma vez que durante as visitas domiciliares é possível a identificação de demandas das famílias cadastradas na ESF sob sua responsabilidade e compartilhar com a equipe de saúde.

Durante os relatos dos ACS, estes reconhecem a necessidade de apoio dos profissionais das ESF para com os familiares e idosos, em especial àqueles dependentes de cuidado, sendo descrito que durante as visitas domiciliares eles percebem que os cuidadores desenvolvem várias atividades diárias além do cuidado ao idoso e, várias vezes, deixam de cuidar de si mesmos. Isso foi evidenciado na sua prática profissional, quando os cuidadores agendam consultas nas ESF com algum profissional e os mesmos faltam nestas, relatando não ter com quem deixar o idoso, 
não poder deixar o idoso sozinho ou por muito tempo com outra pessoa, devido à preocupação e possível demora do atendimento.

Ao final das atividades, os cuidadores, os enfermeiros das ESF, gestor e os ACS sugeriram a realização de outras atividades como essas, especialmente com destaque para às orientações sobre cuidados aos idosos no domicílio, com o propósito de informar/orientar os cuidadores, podendo ser um curso, oficina, um momento de educação em saúde. Essa demanda levantada pelos participantes precisa ser considerada pelos responsáveis pelo cuidado ao idoso, assim como pelos pesquisadores. Na percepção das facilitadoras, a participação conjunta dos profissionais das ESF e cuidadores foi um dos aspectos favorável da devolução dos resultados da pesquisa, isso porque foram expressas algumas angústias e preocupações vivenciadas por cuidadores no domicílio após a assunção da responsabilidade pelo cuidado ao idoso. Também, este momento, como parte do compromisso do pesquisador, oportunizou a troca de saberes e experiências.

\section{DISCUSSÃO}

Em uma pesquisa desenvolvida sobre o retorno do conhecimento sistematizado à comunidade, questiona: o que retornar à comunidade? Qual a melhor forma de fazê-lo? A elaboração da proposta de retorno do conhecimento à comunidade precisa partir de quem? A proposta deve estar clara já na elaboração do projeto ou pode ser elaborada durante a convivência com a comunidade? Esta cita algumas formas de devolução dos resultados da pesquisa à comunidade como por meio de manuais, cartilhas, palestras e cursos. Também demonstra que o retorno dos dados aos participantes da pesquisa é uma das formas de reconhecimento pela ajuda e pelo tempo despendido, e, particularmente, pelo compartilhamento do saber (PATZLAFF; PEIXOTO, 2009). Com isso, verifica-se a importância da realização de estudos sobre a experiência da devolução dos resultados das pesquisas à comunidade como parte da responsabilidade do pesquisador.

A Resolução no 466, de 12 de dezembro de 2012, ao referir sobre os aspectos éticos da pesquisa envolvendo seres humanos, estabelece que as pesquisas devem atender aos fundamentos éticos e científicos pertinentes, entre eles: 1) "garantir que as pesquisas em comunidades, sempre que possível, traduzir-se-ão em benefícios cujos efeitos continuem a se fazer sentir após sua conclusão". "Quando, no interesse da comunidade, houver benefício real em incentivar ou estimular mudanças de costumes ou comportamentos, o protocolo de pesquisa deve incluir, 
sempre que possível, disposições para comunicar tal benefício às pessoas e/ou comunidades"; 2) "comunicar às autoridades competentes, bem como aos órgãos legitimados pelo Controle Social, os resultados e/ou achados da pesquisa, sempre que estes puderem contribuir para a melhoria das condições de vida da coletividade, preservando, porém, a imagem e assegurando que os participantes da pesquisa não sejam estigmatizados”(BRASIL, 2012).

Ainda, a mesma Resolução $\mathrm{n}^{\circ}$ 466, descreve que é necessário: 3) "assegurar aos participantes da pesquisa os benefícios resultantes do projeto, seja em termos de retorno social, acesso aos procedimentos, produtos ou agentes da pesquisa"; e, 4) "assegurar aos participantes da pesquisa as condições de acompanhamento, tratamento, assistência integral e orientação, conforme o caso, enquanto necessário, inclusive nas pesquisas de rastreamento" e, 5) "encaminhar os resultados da pesquisa para publicação, com os devidos créditos aos pesquisadores associados e ao pessoal técnico integrante do projeto" (BRASIL, 2012).

As referir sobre as pesquisas, não se deve esquecer de que o produto do conhecimento é relevante principalmente quando vinculado a um benefício social, ou seja, não somente sob a ótica do que se produz, mas de como este poderá ser consumido (OLIVEIRA, 2010). Neste sentido, após a realização de uma pesquisa, é fundamental que o pesquisador apresente os resultados de seu estudo aos participantes da pesquisa e outros indivíduos envolvidos, para que o saber construído seja compartilhado.

Ademais, nota-se que as atividades de restituição dos resultados à comunidade, mesmo sendo propostas e acordadas no início da pesquisa, precisam ser flexíveis, possibilitando, assim, a inclusão de novas demandas que surgem durante a execução da pesquisa. Isto porque cada comunidade tem suas características próprias e precisam ser consideradas no momento da elaboração da proposta de retorno do saber construído, assim, torna-se complexo estabelecer $a$ priori uma metodologia específica. Entretanto, é imprescindível apontar-se, no planejamento e na apresentação da proposta, possíveis caminhos a serem buscados para que a comunidade também aprenda com a pesquisa realizada (PATZLAFF; PEIXOTO, 2009).

Estudo mostra que, orientado pelo compromisso ético e político, o conhecimento construído por profissionais precisa ser fortalecido socialmente e romper com os muros da academia. Assim, por meio de uma prática crítica e com propostas, será possível influenciar nas condições de vida das pessoas. Também demonstra que a preocupação com o retorno das informações e alcance social das produções refere-se à intenção de fazer o caminho de volta, ou seja, de retornar à realidade que sustentou a produção do conhecimento construído e mobilizar ações que possam modificar realidades (BOURGUIGNON, 2007). 
Devolutiva dos resultados de pesquisa desenvolvida com cuidadores familiares de idosos dependentes

O estudo de Pessanha, Silva e Rotenberg (2013) apresenta que os pesquisadores restituíram os resultados da pesquisa aos participantes, de modo que se apropriem desses, como forma de propiciar o diálogo entre os saberes da experiência e da academia, buscando ampliar os conhecimentos e a capacidade de interferência dos trabalhadores para melhorar suas condições de saúde e trabalho, assim como parte da produção do saber e compromisso ético com os participantes da pesquisa. Este também é um momento de criação de parcerias entre participantes do estudo e pesquisadores.

Sendo assim, o momento da devolutiva dos resultados de pesquisas proporciona a reflexão entre os envolvidos e serve de estratégia para sanar dúvidas, expressar sentimentos, informar demandas e integrar com outras pessoas. Também possibilita a troca de saberes e de experiências, além de favorecer a construção de propostas de intervenção e de novos estudos para serem desenvolvidos a partir das discussões realizadas.

\section{CONSIDERAÇÕES FINAIS}

A partir dessa experiência identificou-se que a devolutiva dos resultados da pesquisa à comunidade é fundamental e parte integrante do compromisso do pesquisador após o desenvolvimento de pesquisas, uma vez que esta prática favoreceu a conciliação entre a produção e a (re)construção de conhecimentos entre os envolvidos no processo de pesquisa e restituição dos resultados. Essa prática ainda possibilitou a troca de saberes e o diálogo sobre os achados do estudo com os diversos atores sociais, como aconteceu durante as atividades realizadas com os cuidadores de idosos, enfermeiros e ACS.

Fica, portanto, explicitada a necessidade da comunidade acadêmica de se comprometer em devolver os resultados aos participantes da pesquisa, uma vez que esta prática irá beneficiálos, assim como contribuir para com o planejamento de ações direcionadas a essas pessoas. Além disso, sugere que essa restituição motiva o desenvolvimento de outras pesquisas, sobretudo, a partir das demandas identificadas no momento da atividade.

Assim, o compartilhamento de experiências foi essencial e ratifica o compromisso dos pesquisadores. Diante do déficit de orientações dos cuidadores familiares evidenciadas na pesquisa de dissertação de mestrado, demonstradas também durante a devolução dos resultados, a partir de dúvidas e questionamentos feitos, sugere-se a continuidade da pesquisa no município com a implementação de ações de educação em saúde aos cuidadores sobre cuidados aos idosos dependentes. 
Devolutiva dos resultados de pesquisa desenvolvida com cuidadores familiares de idosos dependentes

\section{REFERÊNCIAS}

ANJOS, Karla Ferraz dos. Qualidade de vida do familiar cuidador de idosos dependentes no domicílio. 2013. Dissertação (Mestrado). Programa de Pós-Graduação em Enfermagem e Saúde, área de concentração em Saúde Pública. Universidade Estadual do Sudoeste da Bahia - UESB. Jequié, Bahia.

ANJOS, Karla Ferraz dos, BOERY, Rita Narriman Silva de Oliveira, PEREIRA, Rafael.

Qualidade de vida de cuidadores familiares de idosos dependentes no domicílio. Texto Contexto Enferm. v. 23, n. 3, p. 600-8, 2014.

BOURGUIGNON, Jussara Ayres. A particularidade histórica da pesquisa no Serviço Social. Revista Katálysis. v.10, n. (esp), p. 46-54, 2007.

BRASIL. Ministério da Saúde. Secretaria de Atenção à Saúde. Departamento de Atenção Básica. Envelhecimento e saúde da pessoa idosa. 2007.

BRASIL. Ministério da Saúde. Secretaria de Atenção à Saúde, Secretaria de Gestão do Trabalho e da Educação na Saúde. Guia prático do cuidador. Normas e Manuais Técnicos, Série A, 64 p. Brasília: Ministério da Saúde. 2008a.

BRASIL. Ministério da Saúde. Secretaria Especial dos Direitos Humanos, Subsecretaria de Promoção e Defesa dos Direitos Humanos. Cuidar melhor e evitar a violência: Manual do Cuidador da Pessoa Idosa. Brasília. 2008b.

BRASIL. Ministério da Saúde. Conselho Nacional de Saúde. Resolução $\mathbf{n}^{\circ}$ 466, de 12 de dezembro de 2012. 2012.

COREN-BA - Conselho Regional de Enfermagem da Bahia. Principais legislações para o exercício da Enfermagem. Código de Ética dos Profissionais de Enfermagem. Salvador: CRE; 2012.

OLIVEIRA, Adriana Cristina. A trajetória do pesquisador em enfermagem. Editorial. Rev Min Enferm. v. 14, n. 1, p. 11-8, 2010. 
Devolutiva dos resultados de pesquisa desenvolvida com cuidadores familiares de idosos dependentes

PATZLAFF, Rubia Graciela; PEIXOTO, Ariane Luna. A pesquisa em etnobotânica e o retorno do conhecimento sistematizado à comunidade: um assunto complexo. História, Ciências, Saúde-Manguinhos. v. 16, n. 1, p. 237-46, 2009.

PESSANHA, Joseane; SILVA, Claudia Osorio da; ROTENBERG, Lúcia. Uma experiência de restituição de resultados em saúde do trabalhador. Estudos Contemporâneos da Subjetividade. v. 3, n. 1, p. 32-44, 2013. 